\title{
Comment on "An Approximation to Solution of Space and Time Fractional Telegraph Equations by He's Variational Iteration Method"
}

\author{
Yi-Hong Wang ${ }^{1,2}$ and Lan-Lan Huang ${ }^{3}$ \\ ${ }^{1}$ Department of Computer Science, Shanghai Normal University Tianhua College, Shanghai 201815, China \\ ${ }^{2}$ Department of Mathematics, Zhejiang Forestry University, Hangzhou 311300, China \\ ${ }^{3}$ College of Mathematics \& Information Science, Neijiang Normal University, Neijiang 641112, China
}

Correspondence should be addressed to Lan-Lan Huang; mathlan@126.com

Received 7 January 2013; Revised 16 February 2013; Accepted 20 February 2013

Copyright (c) 2013 Y.-H. Wang and L.-L. Huang. This is an open access article distributed under the Creative Commons Attribution License, which permits unrestricted use, distribution, and reproduction in any medium, provided the original work is properly cited.

The variational iteration method was applied to the time fractional telegraph equation and some variational iteration formulae were suggested in (Sevimlican, 2010). Those formulae are improved by Laplace transform from which the approximate solutions of higher accuracies can be obtained.

Sevimlican [1] considered the application of the variational iteration method $[2,3]$ to find approximate solutions of space and time fractional telegraph equations. The author suggested the following variational iteration formula for (5.1) in [1]

$$
\begin{array}{r}
u_{n+1}=u_{n}+\int_{0}^{x} \lambda(s, x)\left(\frac{\partial^{\alpha} u(s, t)}{\partial s^{\alpha}}-\frac{\partial^{2} u(s, t)}{\partial t^{2}}\right. \\
\left.-\frac{\partial u(s, t)}{\partial t}-u(s, t)\right) d s, \\
1<\alpha<2,
\end{array}
$$

$$
\lambda(s, x)=s-x .
$$

However, in this comment, it is pointed out that the identification of the Lagrange multiplier $\lambda(s, x)=s-x$ from (4.1) to (4.9) can be improved.

According to the technique of determination of the Lagrange multipliers $[4,5]$, firstly, construct a correctional functional as

$$
\begin{aligned}
& u_{n+1}(x, t) \\
& =u_{n}(x, t) \\
& \quad+\int_{0}^{x} \lambda(s, x)\left(\frac{\partial^{\alpha} u_{n}(s, t)}{\partial s^{\alpha}}-\frac{\partial^{2} u_{n}(s, t)}{\partial t^{2}}\right. \\
& \left.\quad-\frac{\partial u_{n}(s, t)}{\partial t}-u_{n}(s, t)\right) d s, \quad \alpha>0 .
\end{aligned}
$$

Assuming the Lagrange multiplier $\lambda(s, x)=\lambda(x-s)$, take the Laplace transform to both sides of (2)

$$
\begin{aligned}
\bar{u}_{n+1}(S, t)=\bar{u}_{n}(S, t) & \\
+L\left[\int_{0}^{x} \lambda(s, x)(\right. & \frac{\partial^{\alpha} u_{n}(s, t)}{\partial s^{\alpha}}-\frac{\partial^{2} u_{n}(s, t)}{\partial t^{2}} \\
& \left.\left.-\frac{\partial u_{n}(s, t)}{\partial t}-u_{n}(s, t)\right) d s\right],
\end{aligned}
$$

where $\bar{u}_{n}(S, t)$ is the Laplace transform of $u_{n}(x, t)$.

Taking the variation $\delta$ with respect to $\bar{u}_{n}(S, t)$, one can obtain

$$
\begin{aligned}
\delta \bar{u}_{n+1}(S, t)= & \delta \bar{u}_{n}(S, t) \\
& +\delta L\left[\int _ { 0 } ^ { x } \lambda ( s , x ) \left(\frac{\partial^{\alpha} u_{n}(s, t)}{\partial s^{\alpha}}-\frac{\partial^{2} u_{n}(s, t)}{\partial t^{2}}\right.\right. \\
& \left.\left.-\frac{\partial u_{n}(s, t)}{\partial t}-u_{n}(s, t)\right) d s\right] \\
= & \left(1+\bar{\lambda}(S) S^{\alpha}\right) \delta \bar{u}_{n}(S, t), \\
\bar{\lambda}(S)= & -\frac{1}{S^{\alpha}} .
\end{aligned}
$$


Then, the Lagrange multiplier can be determined as

$$
\lambda(s, x)=\frac{(-1)^{\alpha}(s-x)^{\alpha-1}}{\Gamma(\alpha)} .
$$

Instead $\lambda(s, x)=s-x($ see $(4.9)$ in [1]).

As a result, the variational iteration formula is obtained as

$$
\begin{array}{r}
u_{n+1}=u_{n}+\int_{0}^{x} \frac{(-1)^{\alpha}(s-x)^{\alpha-1}}{\Gamma(\alpha)}\left(\frac{\partial^{\alpha} u(s, t)}{\partial s^{\alpha}}-\frac{\partial^{2} u(s, t)}{\partial t^{2}}\right. \\
\left.-\frac{\partial u(s, t)}{\partial t}-u(s, t)\right) d s \\
0<\alpha .
\end{array}
$$

The variational iteration formulae (5.10) and (5.17) are not right which also should be corrected, respectively.

Equation (5.10) in [1] should be

$$
\begin{array}{r}
u_{n+1}=u_{n}+\int_{0}^{x} \frac{(s-x)^{2 \alpha-1}}{\Gamma(2 \alpha)}\left(\frac{\partial^{2 \alpha} u(s, t)}{\partial s^{2 \alpha}}-\frac{\partial^{2} u(s, t)}{\partial t^{2}}\right. \\
-\frac{\partial u(s, t)}{\partial t}-u(s, t)+s^{2} \\
+t-1) d s, \quad 0<\alpha .
\end{array}
$$

Equation (5.17) in [1] should be

$$
\begin{array}{r}
u_{n+1}=u_{n}+\int_{0}^{t} \frac{(s-t)^{2 \alpha-1}}{\Gamma(2 \alpha)}\left(\frac{\partial^{2 \alpha} u(x, s)}{\partial s^{2 \alpha}}+\lambda \frac{\partial^{2} u(x, s)}{\partial s^{2}}\right. \\
\left.-v \frac{\partial u(x, s)}{\partial s}\right) d s, \quad 0<\alpha .
\end{array}
$$

\section{Conclusions}

As is well known, the VIM became an efficient analytical tool in nonlinear science since it was proposed and the method was often used in fractional differential equations. This study illustrates the method in fractional calculus can be improved by the Laplace tranform method with which the Lagrange mutipliers can be identified explicitly.

Recently, there are also other new applications of the variation iteration method to various nonlinear problems, that is, fuzzy equations $[6,7]$ and $q$-fractional differential equations [8]. Readers are referred to the recent review article [9].

\section{Acknowledgment}

This work is financially supported by the Zhejiang Natural Science Foundation (Grant no. LQ12A01010).

\section{References}

[1] A. Sevimlican, "An approximation to solution of space and time fractional telegraph equations by He's variational iteration

method," Mathematical Problems in Engineering, vol. 2010, Article ID 290631, 10 pages, 2010.

[2] J. H. He, "Approximate analytical solution for seepage flow with fractional derivatives in porous media," Computer Methods in Applied Mechanics and Engineering, vol. 167, no. 1-2, pp. 57-68, 1998.

[3] J. H. He, "Variational iteration method-a kind of non-linear analytical technique: some examples," International Journal of Non-Linear Mechanics, vol. 34, no. 4, pp. 699-708, 1999.

[4] G. C. Wu, "Variational iteration method for solving the timefractional diffusion equations in porous medium," Chinese Physics B, vol. 21, no. 12, Article ID 120504, 2012.

[5] G. C. Wu and D. Baleanu, "Variational iteration method for the Burgers' flow with fractional derivatives-new Lagrange multipliers," Applied Mathematical Modelling, vol. 37, no. 9, pp. 6183-6190, 2013.

[6] H. Jafari, M. Saeidy, and D. Baleanu, "The variational iteration method for solving $n$-th order fuzzy differential equations," Central European Journal of Physics, vol. 10, no. 1, pp. 76-85, 2012.

[7] H. Jafari and C. M. Khalique, "Homotopy perturbation and variational iteration methods for solving fuzzy differential equations," Communications in Fractional Calculus, vol. 3, no. 1, pp. 38-48, 2012.

[8] G. C. Wu and D. Baleanu, "New applications of the variational iteration method-from differential equations to $q$-fractional difference equations," Advances in Difference Equations, vol. 2013, article 21, 2013.

[9] J. H. He, "Asymptotic methods for solitary solutions and compactons," Abstract and Applied Analysis, vol. 2012, Article ID 916793, 130 pages, 2012. 


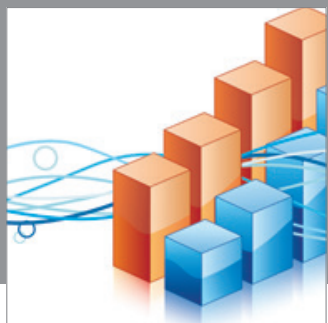

Advances in

Operations Research

mansans

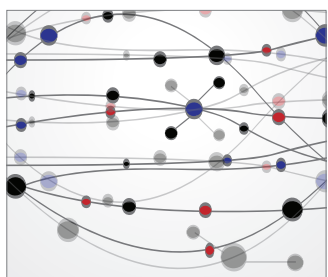

The Scientific World Journal
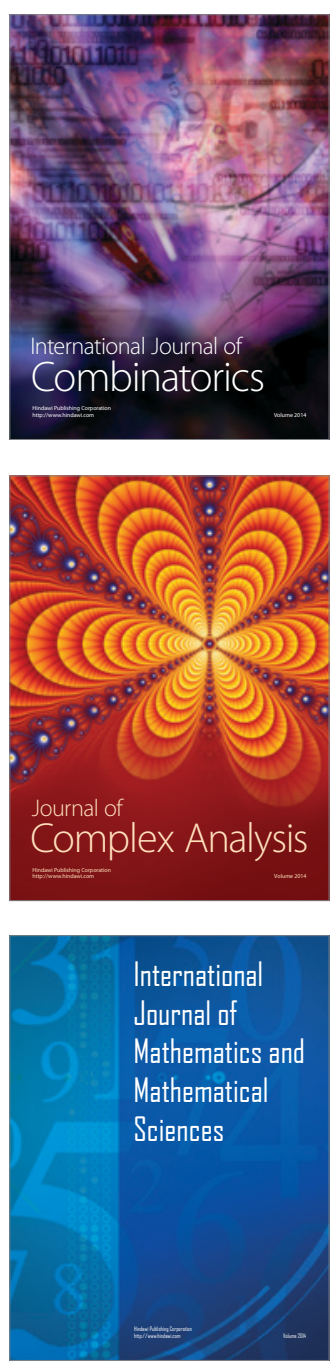
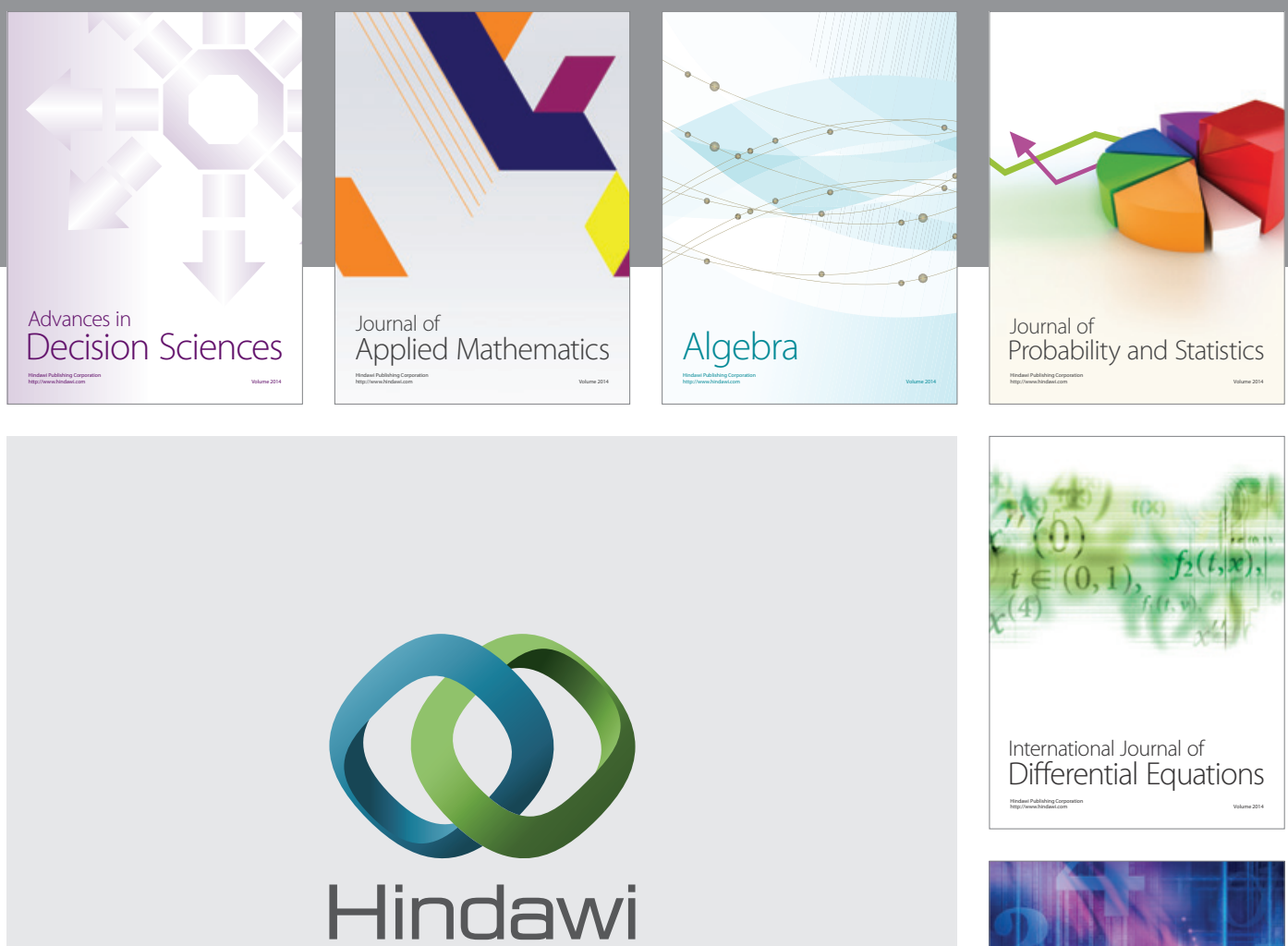

Submit your manuscripts at http://www.hindawi.com
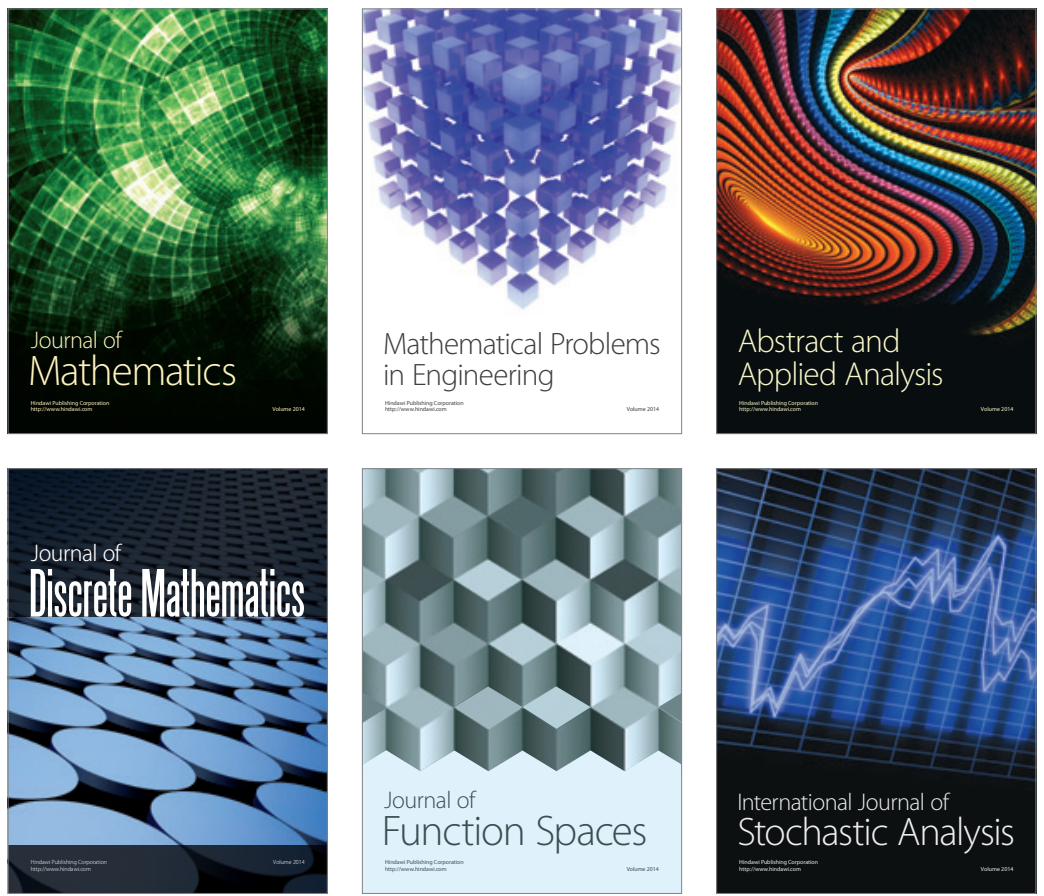

Journal of

Function Spaces

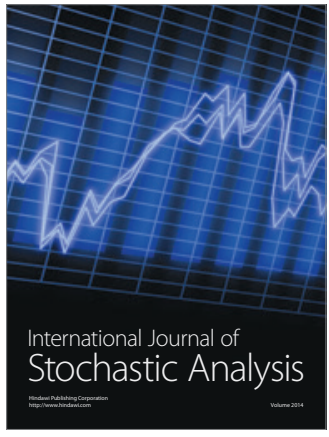

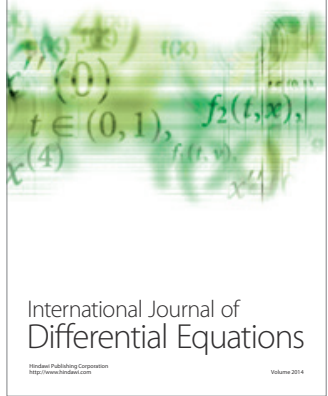
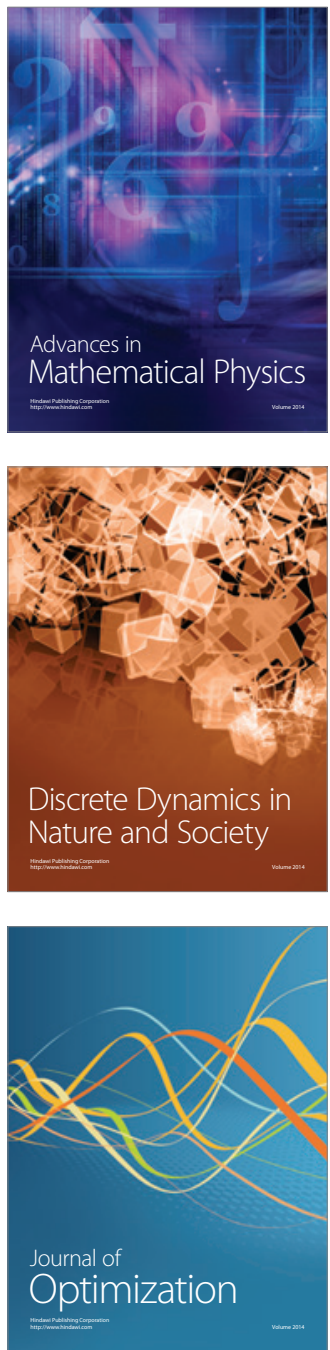